\title{
Branching of negative streamers in free flight
}

\author{
Andrea Rocco, ${ }^{1}$ Ute Ebert, ${ }^{1,2}$ and Willem Hundsdorfer ${ }^{1}$ \\ ${ }^{1}$ Centrum voor Wiskunde en Informatica, P.O. Box 94079, 1090 GB Amsterdam, The Netherlands \\ ${ }^{2}$ Department of Physics, TU Eindhoven, $5600 \mathrm{MB}$ Eindhoven, The Netherlands
}

(Received 22 April 2002; published 24 September 2002)

\begin{abstract}
We have recently shown that a negative streamer in a sufficiently high homogeneous field can branch spontaneously due to a Laplacian instability, rather than approach a stationary mode of propagation with fixed radius. In our previous simulations, the streamer started from a wide initial ionization seed on the cathode. We here demonstrate, in improved simulations, that a streamer emerging from a single electron branches in the same way. In fact, though the evolving streamer is much more narrow, it branches after an even shorter propagation distance.
\end{abstract}

DOI: 10.1103/PhysRevE.66.035102

PACS number(s): 82.40.Ck, 05.45.-a, 47.54.+r, 52.80.-s

Streamers are widely known phenomena in electric breakdown: long and rapidly growing channels of high ionization penetrating a region of low or vanishing ionization under the influence of a strong electric field [1,2]. In experiments, streamers frequently are seen to branch. On a phenomenological level, the so-called dielectric break-down model [3-5], a variation of diffusion limited aggregation [6], has been suggested as a stochastic process explaining the branching. Such models are inspired by earlier concepts of streamer propagation where the space charge was assumed to be smeared out over the full streamer head. Branching then would occur due to randomly distributed ionization avalanches around the streamer. Such stochastic phenomenological models on larger scale have not been related to particular discharge models.

However, simulations $[7,8]$ have established that the space charge of the streamer is concentrated in a thin layer around the head rather than being smeared out over the full head. This leads to a different field distribution and a much faster propagation mode, and to a revival of the concept of an ideally conducting streamer formulated by Lozansky and Firsov [9]. In particular, this charge distribution allows for a completely different "Laplacian" instability mechanism of streamers $[10,11]$ that can operate even without any randomness quite like in viscous fingering, dendritic growth of solids into undercooled melts, etc. It naturally emerges in the minimal streamer model (1)-(4). In a recent paper [12], we found that in contrast to previous expectations, this Laplacian instability mechanism can cause the spontaneous branching of streamers propagating in a strong homogeneous field. The system was identical to that of previous simulations $[7,8]$ except that the field was twice as high. However, our simulation results were somewhat limited by numerical constraints. Because of the unexpected results [13] and some nonsmooth structures in the figures, some researchers wondered whether the figures showed a numerical rather than a physical instability [14].

Therefore, we here present simulations with improved numerics, and we show more details of the evolution. In particular, we increased the number of grid points in the simulations from $1000 \times 1000$ to $2000 \times 2000$, which improves both the accuracy of the numerical output data and the qual- ity of the contour plots, so the plots are smooth. Furthermore, we changed the boundary condition on the cathode from homogeneous Neumann $\partial_{z} \sigma=0$ to homogeneous Dirichlet $\sigma$ $=0$ for the electron density $\sigma$. This means that while previously the electrons could flow freely from the metal of the electrode into the gas, this current is now suppressed. With these improvements, the choice of the initial conditions now ceases to be constrained by numerical considerations. We continue to use cylinder symmetry to calculate effectively on a two-dimensional grid. Doing so, we keep relying on the analytical argument that the constraint of cylindrical geometry suppresses some instability modes, and that therefore a truly three-dimensional system would become unstable earlier or at the same time as the system with symmetry constraint, but certainly not later.

The improvements of the numerical code allow now for the simulation of a streamer starting from a single electron on the cathode rather than from a wide initial seed as in Ref. [12]. Our new figures show the streamer branch in free flight. The initial electron on the cathode creates first an avalanche, then a streamer, and finally the streamer splits. The situation resembles the historical experiments of Raether [1], except that the field is higher.

In detail, we investigate the minimal streamer model, i.e., a "fluid approximation" with local field-dependent impact ionization reaction in the Townsend approximation [2] in a nonattaching and nonionized gas. In dimensionless units [10-12], the model has the form

$$
\begin{gathered}
\partial_{t} \sigma-\nabla \cdot(\sigma \mathbf{E}+D \boldsymbol{\nabla} \sigma)=\sigma f(|\mathbf{E}|), \\
\partial_{t} \rho=\sigma f(|\mathbf{E}|), \\
\rho-\sigma=\nabla \cdot \mathbf{E}, \quad \mathbf{E}=-\nabla \Phi, \\
f(|\mathbf{E}|)=|\mathbf{E}| e^{-1 /|\mathbf{E}|} .
\end{gathered}
$$

Here $\sigma$ and $\rho$ are the densities of electrons and positive ions, $\mathbf{E}$ is the electric field, $\boldsymbol{\Phi}$ is the electric potential, and $D$ the dimensionless diffusion constant. The mobility of ions is neglected. The same model was investigated in Refs. [7,8]. The translation to physical units depends on the type and the 

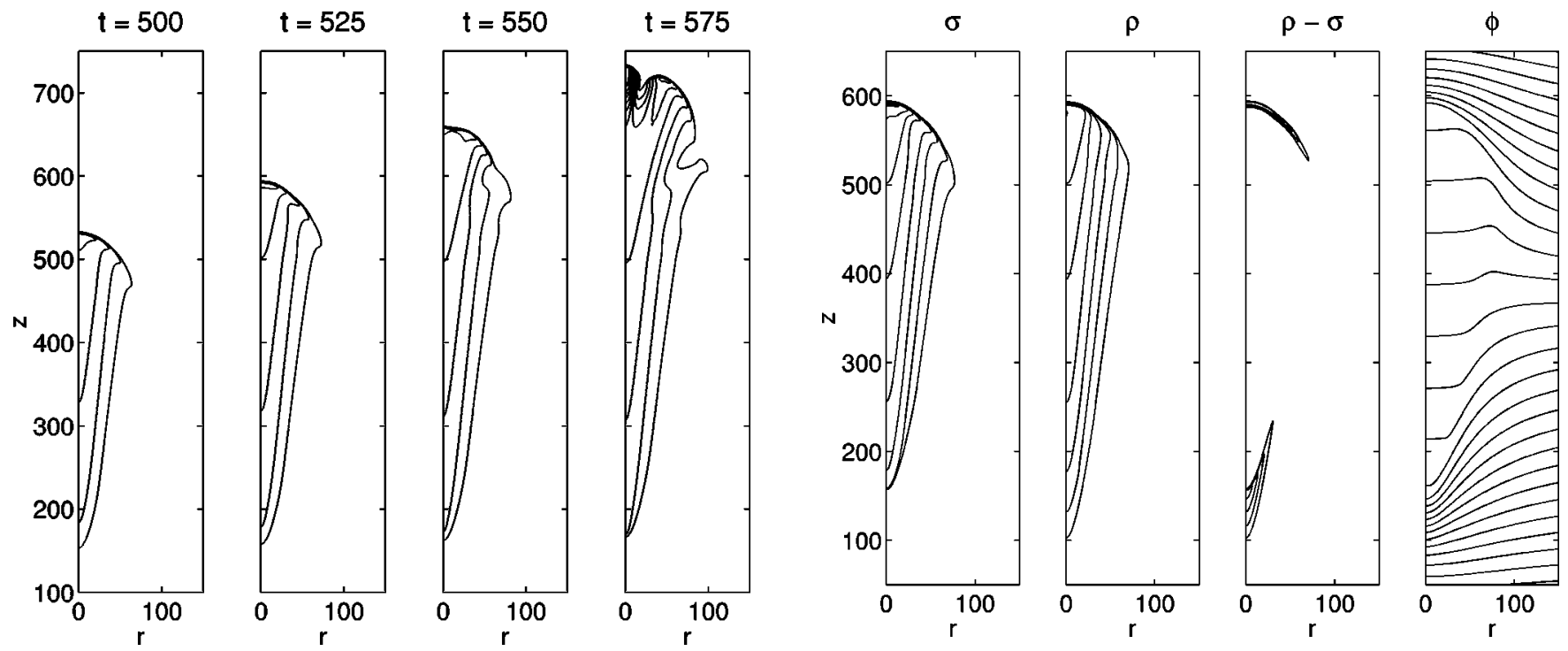

FIG. 1. Evolution of an anode directed streamer in a strong homogeneous background field. The planar cathode is located at $z$ $=0$ and the planar anode at $z=2000$. The radial coordinate extends from the origin up to $r=2000$ to assure homogeneous field conditions. Shown is $100 \leqslant z \leqslant 750$ and $0 \leqslant r \leqslant 150$ with equal axis scaling. The thin lines denote levels of equal electron density $\sigma$ with increments of 0.15 . All plotted quantities are dimensionless.

pressure $p$ of the gas. With an effective field-dependent impact ionization coefficient $\alpha(E)=A p \exp (-B p /|E|)$ and electron mobility $\mu_{e}=\bar{\mu} / p$ as in Ref. [2], and with the parameter values for nitrogen as in Refs. [7,8], we get for the scales of length, time, field, and particle density,

$$
\begin{gathered}
l_{0} \simeq \frac{2.3 \mu \mathrm{m}}{(p / 1 \mathrm{bar})}, \quad t_{0} \simeq \frac{3 \mathrm{ps}}{(p / 1 \mathrm{bar})}, \quad E_{0} \simeq 200 \frac{\mathrm{kV}}{\mathrm{cm}} \frac{p}{1 \mathrm{bar}}, \\
n_{0} \simeq \frac{5 \times 10^{14}}{\mathrm{~cm}^{3}}\left(\frac{p}{1 \mathrm{bar}}\right)^{2} \simeq 2 \times 10^{-5}\left[N_{2}\right] \frac{p}{1 \mathrm{bar}},
\end{gathered}
$$

where $\left[N_{2}\right]$ is the neutral gas particle density. The pressure dependent scaling relations are of interest for laboratory experiments, as well as for so-called sprite discharges [15] in the mesosphere above thunderclouds.

The simulation is performed with the same voltage of 1000 and electrode distance of 2000 as in Ref. [12], corresponding to a field of $100 \mathrm{kV} / \mathrm{cm}$ and an electrode distance of $4.6 \mathrm{~mm}$ for nitrogen at $p=1$ bar. As an initial condition, one electron was distributed over one cell of the numerical grid next to the cathode. Because of the diffusive broadening of the drifting avalanche, this "distribution" of the initial electron is permissible.

In the figures, we omit the avalanche phase and concentrate on the developed streamer and its branching at times $t$ $=500,525,550$, and 575. The field is applied in the $z$ direction, and $r$ is the radial coordinate extending up to $r=2000$ to suppress artifacts from the lateral boundaries. The simulation shows a streamer not connected to the electrode. It has

FIG. 2. The streamer at time $t=525$. The figures show contour plots with equal aspect ratio for the electron density $\sigma$, ion density $\rho$, space charge density $\rho-\sigma$, and electric potential $\Phi$. The level lines for the densities $\sigma$ and $\rho$ are multiples of 0.1 , for the space charge density $\rho-\sigma$ of 0.05 , and the increment between different levels of the potential is 10 .

the conical shape of the initial avalanche created by a single electron in a homogeneous field as first found in Raether's experiments [1].

Figure 1 shows the electron density $\sigma$ within the whole streamer body at four snapshots of the evolution. Due to the no-current boundary condition on the electrodes, the streamer is not connected to the cathode at $z=0$. The equidensity lines mark multiples of 0.15 in all snapshots. The electrodes at $z=0$ and $z=2000$ are outside the region shown. The qualitative evolution is the same as in our previous paper [12]: while the streamer extends, it becomes wider, the electron density increases, and finally the instability develops.

Figure 2 shows four different observables of the whole streamer at one time instant $t=525$. We plot the electron density $\sigma$, the ion density $\rho$, the space charge density $\rho$ $-\sigma$, and the electric potential $\Phi$. In the propagating front at the tip of the streamer, an overshoot of drifting electrons creates a negatively charged layer, while the back of the streamer is depleted from electrons and is positively charged by the essentially immobile ions left behind. Therefore the "ion streamer" is longer in the back, and the ion density has no overshoot in the propagating tip. The resulting space charge densities are shown in the third plot: a positive space charge with a maximum density of 0.25 in the back, and a negative space charge with a minimum density of -0.25 in the propagating tip. (This coincidence of absolute values is accidental.) While the whole object is electrically neutral, the electric polarization leads to a suppression of the field in the interior. This can be read from the large distance between equipotential lines inside the ionized body visible in the last plot. The streamer is indeed approaching the LozanskyFirsov limit of "ideal conductivity" [9]. 

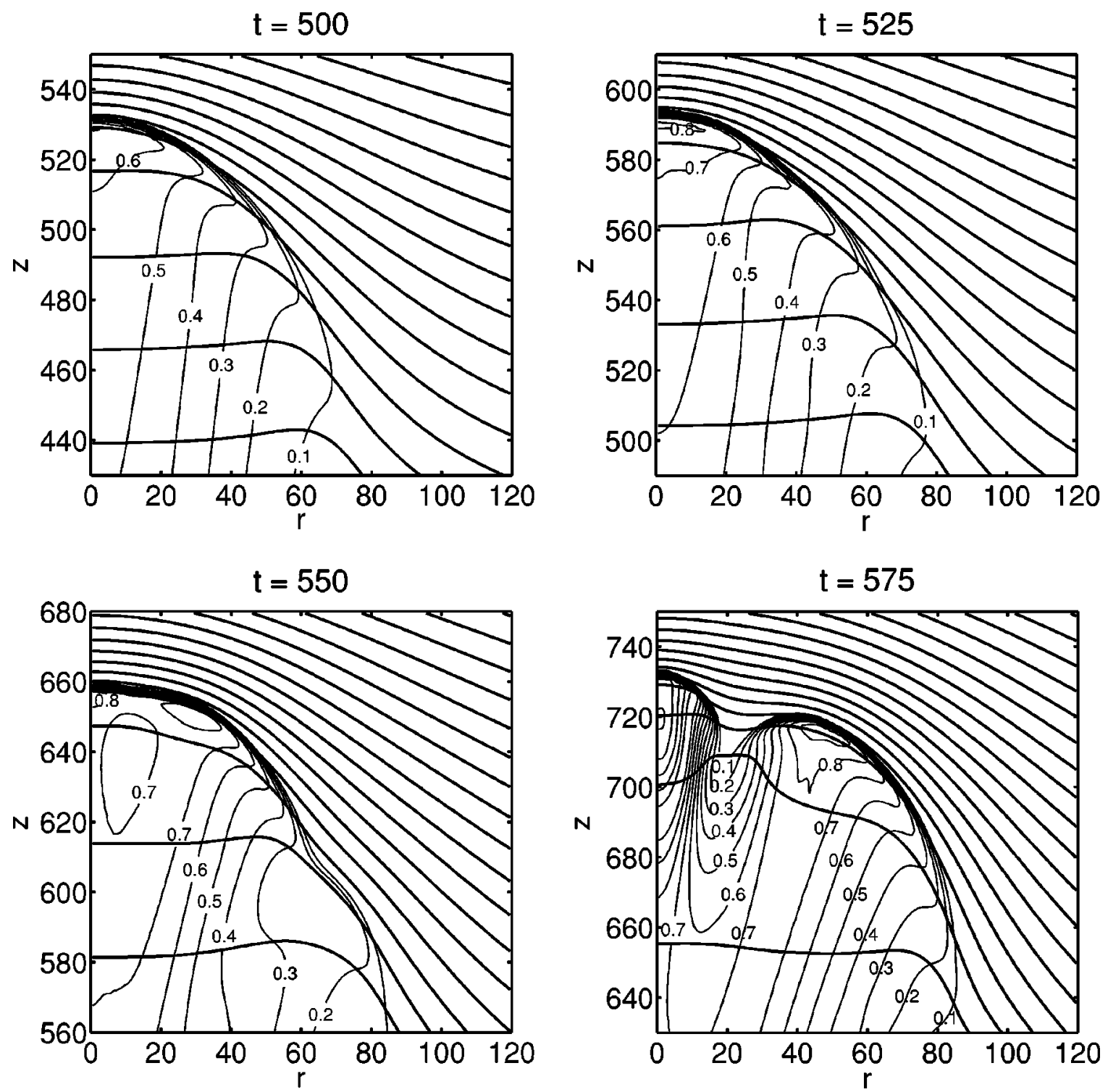

FIG. 3. Enlargement of the evolution of the streamer tip showing electron density $\sigma$ with increments of 0.1 as thin lines and electric potential $\Phi$ with increments of 5 as thick lines. At the last time step $t=575$, the maximal electron density in the core of the finger on the axis is 1.5. All figures have equal aspect ratio and equal axis scaling.

Figure 3 shows an enlargement the tip of the streamer at the same time steps as in Fig. 1. Again the electron densities $\sigma$ are plotted, and addionally the equipotential lines $\Phi$. Already at time $t=525$, the onset of the instability can be concluded from the somewhat irregular shape of the level lines. The curvature of the tip decreases, the densities increase, the field in the interior decreases, and the field ahead of the streamer increases. Consequently, the tip becomes unstable and splits.

We conclude with a few remarks:

(1) It is quite remarkable that the finger of the proposed simulations branches after an even shorter travel distance than the one growing out of a wide, highly ionized seed in Ref. [12], though it is more narrow during its whole evolution.

(2) When the initial electron is not placed on the electrode but somewhere in the gap, simulations show the same figures as in this paper, essentially unchanged except for a trivial shift in space.

(3) The transition times from avalanche to streamer and then further from streamer into the instability depend quite nonlinearly on the externally applied field, as we will discuss in a forthcoming paper; therefore the question whether branching also would occur in a lower field after a longer propagation distance is open.

(4) The discovery of sprite discharges [15] in the mesosphere gives a new impetus to the study of negative streamers in high fields, since a negative sprite discharge propagates into increasing heights, i.e., areas of decreasing pressure; the scaling laws (5) therefore imply that the effective field continues to grow along their path.

(5) Sprite discharges, on the other hand, revive the interest 
in the role of preionization as studied in Ref. [7]. Preionization also for a while has been advocated as a substitute for nonlocal photoionization that is important in particular in composite gases such as air; however, this substitution appears fairly unsystematic. Both preionization and photoionization make the ionization front smoother and therefore more stable, so the tip splitting instability will set in later. At the same time, they increase the propagation velocity of a front in a given field, and they decrease the demands on the spatial resolution of the simulation.

A.R. was supported by the Dutch Research School CPS, the Dutch Physics Funding Agency FOM, and CWI Amsterdam.
[1] H. Raether, Z. Phys. 112, 464 (1939) (in German).

[2] Y. P. Raizer, Gas Discharge Physics, 2nd ed. (Springer, Berlin, 1991, 1997).

[3] L. Niemeyer, L. Pietronero, and H.J. Wiesmann, Phys. Rev. Lett. 52, 1033 (1984).

[4] A.D.O. Bawagan, Chem. Phys. Lett. 281, 325 (1997).

[5] V.P. Pasko, U.S. Inan, and T.F. Bell, Geophys. Res. Lett. 28, 3821 (2001).

[6] T.A. Witten and L.M. Sander, Phys. Rev. Lett. 47, 1400 (1981).

[7] S.K. Dhali and P.F. Williams, Phys. Rev. A 31, 1219 (1985); J. Appl. Phys. 62, 4696 (1987).

[8] P.A. Vitello, B.M. Penetrante, and J.N. Bardsley, Phys. Rev. E 49, 5574 (1994).

[9] E.D. Lozansky and O.B. Firsov, J. Phys. D 6, 976 (1973).

[10] U. Ebert, W. van Saarloos, and C. Caroli, Phys. Rev. Lett. 77, 4178 (1996); Phys. Rev. E 55, 1530 (1997).
[11] U. Ebert and M. Arrayás, in Coherent Structures in Complex Systems, edited by D. Reguera, et al., Lecture Notes in Physics Vol. 567 (Springer, Berlin 2001), pp. 270-282 .

[12] M. Arrayás, U. Ebert, and W. Hundsdorfer, Phys. Rev. Lett. 88, 174502 (2002).

[13] P. Ball, Lightning Forks Illuminated, science update in Nature, April 10 2002, http://www.nature.com/nsu/020408/0204084.html

[14] P. R. Minkel, Sparks Branch Like Coral Reefs, Physical Review Focus, April 162002 accompanying [12], http:// focus.aps.org/v9/st19.html

[15] V.P. Pasko, U.S. Inan, and T.F. Bell, Geophys. Res. Lett. 25, 2123 (1998); E.A. Gerken, U.S. Inan, and C.P. BarringtonLeigh, ibid. 27, 2637 (2000); V.P. Pasko, M.A. Stanley, J.D. Mathews, U.S. Inan, and T.G. Wood, Nature (London) 416, 152 (2002). 